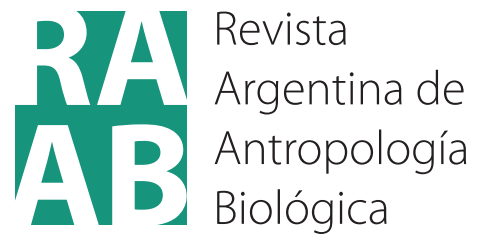

TRABAJO ORIGINAL

\section{Primer estudio paleoparasitológico en restos humanos de la provincia de Córdoba (Holoceno tardío)}

\section{First paleoparasitological study on human remains from the province of Córdoba (late Holocene)}

ANTROPOLOGÍA BIOLÓGICA

Volumen 23, Número 1

Enero-Junio 2021

Financiamiento: Agencia Nacional de Promoción Científica y Tecnológica, PICT 2013-2028 y 2015-3155. Secretaría de Ciencia y Técnica UNC, 2016-2017. Consejo Interuniversitario Nacional, Res. P. N³18/15 y 356/16. Conselho Nacional de Desenvolvimento Científico e Tecnológico, 307932/2014$1,312934 / 2017$ y $151879 / 2019-2$. Coordenação de Aperfeiçoamento de Pessoal de Nível Superior, 847/15. Fundação Carlos Chagas Filho de Amparo à Pesquisa do Estado do Rio de Janeiro CNE E-26/202.945/2016 y E-26/010.000165/2016.

*Correspondencia a: Alena Mayo Iñiguez. Laboratorio de Biología de Tripanosomatídeos, Instituto Oswaldo Cruz, Fundación Oswaldo Cruz (LABTRIPIOC-FIOCRUZ). Av. Brasil 4365, Río de Janeiro, Río de Janeiro, Brasil. E-mail: alenainiguez@gmail.com; a: Mariana Fabra. Instituto de Antropología de Córdoba IDACOR (UNC/CONICET).

Consejo Nacional de Investigaciones Científicas y Técnicas. Museo de Antropología, Facultad de Filosofía y Humanidades, Universidad Nacional de Córdoba. Av. Hipólito Yrigoyen 174, Córdoba, Córdoba, Argentina. E-mail: marianafabra@gmail.com

RECIBIDO: 18 Julio 2020

ACEPTADO: 9 Octubre 2020

DOI: $10.24215 / 18536387 e 030$

e-ISSN 1853-6387

https://revistas.unlp.edu.ar/raab

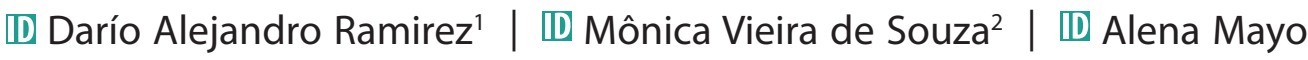 \\ Iñiguez $^{3 *} \mid$ 四 Mariana Fabra ${ }^{4}$
}

1) Instituto de Antropología de Córdoba IDACOR (UNC/CONICET). Museo de Antropología, Facultad de Filosofía y Humanidades, Universidad Nacional de Córdoba. Av. Hipólito Yrigoyen 174, Córdoba, Córdoba, Argentina. 2) Laboratorio de Biología de Tripanosomatídeos, Instituto Oswaldo Cruz, Fundación Oswaldo Cruz (IOC-FIOCRUZ). Av. Brasil 4365, Río de Janeiro, Río de Janeiro, Brasil. 3) Laboratorio de Biología de Tripanosomatídeos, Instituto Oswaldo Cruz, Fundación Oswaldo Cruz (LABTRIP-IOC-FIOCRUZ). Av. Brasil 4365, Río de Janeiro, Río de Janeiro, Brasil. 4) Instituto de Antropología de Córdoba IDACOR (UNC/CONICET). Consejo Nacional de Investigaciones Científicas y Técnicas. Museo de Antropología, Facultad de Filosofía y Humanidades, Universidad Nacional de Córdoba. Av. Hipólito Yrigoyen 174, Córdoba, Córdoba, Argentina.

\section{Resumen}

La Paleoparasitología en la Argentina ha producido un gran número de trabajos, especialmente en Patagonia. Sin embargo, hay ausencia de estos estudios en las poblaciones de la región central del país. En este sentido, realizamos la primera investigación sobre parasitosis en restos humanos de sitios arqueológicos de la provincia de Córdoba. Se estudiaron 13 individuos de 4 sitios arqueológicos, datados entre $2562 \pm 47$ y $533 \pm 57{ }^{14} \mathrm{C}$ años AP. Los análisis paleoparasitológicos se aplicaron sobre 20 muestras de sedimentos provenientes de la cavidad pélvica, empleando las técnicas de sedimentación espontánea, disgregación en ácido clorhídrico y flotación en sacarosa. Se hallaron estructuras sugestivas de huevos de helmintos de las superfamilias Ascaridoidea y Heterakoidea, y otros de nemátodos no identificados. Debido a que los animales son los huéspedes definitivos de estos parásitos, los resultados podrían sugerir la presencia de falso parasitismo en los individuos de la región. La relación cercana de los humanos con los animales, utilizados como parte de la dieta, se sustenta en estudios isotópicos y zooarqueológicos previos. La escasez de hallazgos parasitarios en este estudio posiblemente se debe a factores tafonómicos y a las características ambientales de los sitios. Rev Arg Antrop Biol 23(1), 2021. doi: 10.24215/18536387e030

Palabras Clave: Paleoparasitología; sedimentos arqueológicos; interacciones ecológicas; Heterakoidea; Ascaridoidea 


\begin{abstract}
Paleoparasitology in Argentina has produced a number of studies, especially from Patagonia. However, investigations on parasites in past populations from the central region of the country are absent. In this regard, we performed the first research on parasitosis in human remains from archaeological sites of the province of Córdoba. Thirteen individuals from four archaeological sites, dated of $2562 \pm 47$ to $533 \pm 57{ }^{14} \mathrm{C}$ years BP, were examined. Paleoparasitological analyses were conducted on 20 sediment samples from the pelvic cavity, using spontaneous sedimentation, disaggregation in hydrochloric acid, and sucrose flotation techniques. We found structures suggestive of helminth eggs of Ascaridoidea and Heterakoidea superfamilies, and of unidentified nematode eggs. As animals are the definitive hosts of the parasites found, the results could suggest the occurrence of false parasitism in the individuals from the region. The close interaction between humans and animals, incorporated as part of the diet, is supported by previous isotopic and zooarchaeological studies. The scarcity of parasite findings in this study would be attributed to taphonomic processes and the environmental characteristics of the sites. Rev Arg Antrop Biol 23(1), 2021. doi: 10.24215/18536387e030
\end{abstract}

Keywords: Paleoparasitology; archaeological sediments; ecological interactions; Heterakoidea; Ascaridoidea

La Paleoparasitología es la disciplina que estudia el origen y la evolución de los parásitos en materiales antiguos, tanto de origen arqueológico como paleontológico (Araújo, Reinhard, Ferreira, Pucu y Chieffie, 2013). Las técnicas paleoparasitológicas han permitido detectar parasitosis y, en ocasiones, diagnosticar enfermedades en poblaciones del pasado. Los materiales de estudio incluyen coprolitos, sedimentos asociados a la cavidad pélvica de esqueletos, así como de letrinas, basureros y otros espacios en sitios arqueológicos y, en menor medida, tejidos momificados y bolos de regurgitación de aves rapaces (Fugassa, 2014). Dado a que algunas especies de parásitos necesitan condiciones ambientales específicas para completar sus ciclos biológicos, el hallazgo de restos parasitarios como larvas, huevos y quistes en muestras antiguas permite estimar los escenarios paleoambientales y paleoclimáticos en los que las poblaciones vivieron (Le Bailly et al., 2006), así como los patrones de movilidad o migraciones en el pasado (Araújo, Reinhard, Ferreira y Gardner, 2008). También es posible hacer inferencias sobre la dieta, el estado de salud y las prácticas culturales de grupos humanos antiguos (Yeh, Prag, Clamer, Humbert y Mitchell, 2015).

En Argentina, esta disciplina tuvo un gran desarrollo en los últimos quince años principalmente en Patagonia, pudiendo confirmar la presencia de parasitosis en poblaciones humanas desde la transición Pleistoceno-Holoceno (Araújo et al., 2015; Fugassa, 2006; Fugassa, Beltrame, Sardella, Civalero y Aschero, 2010; Fugassa y Petrigh, 2019; Fugassa, Petrigh, Fernández, Carballido Catalayud y Belleli, 2018; Fugassa, Sardella, Guichón, Denegri y Araújo, 2008). Asimismo, el hallazgo de parásitos en coprolitos de animales y bolos de regurgitación de aves permitió conocer una gran diversidad de especies presentes en el pasado y el riesgo de zoonosis que esto habría implicado para los grupos humanos relacionados (Beltrame, Pruzzo, Sanabria, Pérez y Mora, 2020; Beltrame, Vieira de Souza, Araújo y Sardella, 2014; Tietze, Barberena y Beltrame, 2019).

En la región central de Argentina se han desarrollado líneas de investigación desde una 
perspectiva bioarqueológica, centradas en las formas de vida de las poblaciones humanas que habitaron los diferentes ambientes ecológicos del actual territorio de Córdoba. Estos estudios han revelado persistencias y cambios en el estilo de vida de estas poblaciones durante los últimos 4500 años, específicamente cambios en la frecuencia de algunas patologías y actividades físicas realizadas (Salega y Fabra, 2013), en las estrategias de subsistencia (Fabra y González, 2019; Laguens, Fabra, Dos Santos y Demarchi, 2009; Tavarone, Colobig y Fabra, 2019), y en la estructura biológica y la historia evolutiva de las poblaciones (Fabra y Demarchi, 2013; Nores, Fabra, García y Demarchi, 2017). Sin embargo, hasta la fecha no se habían realizado estudios que permitieran detectar la presencia de parásitos en poblaciones arqueológicas de la región.

En este marco, el objetivo de este trabajo es presentar los resultados de los primeros análisis paleoparasitológicos realizados en individuos de cuatro sitios arqueológicos de la Provincia de Córdoba, fechados en el Holoceno tardío, entre $2562 \pm 47$ y $533 \pm 57{ }^{14} \mathrm{C}$ años AP. Se ha considerado que el nucleamiento social, el crecimiento poblacional y la reducción de la movilidad residencial asociados a las prácticas hortícolas tuvieron lugar alrededor de 1500-1200 años AP. Se propone como hipótesis, que estos procesos, así como la proximidad y la ingesta de ciertos animales silvestres, habrían favorecido el aumento de patologías de origen infeccioso causadas por parásitos. Así, es de esperar la presencia de parásitos gastrointestinales en poblaciones humanas hacia finales del Holoceno tardío.

\section{MATERIALES Y MÉTODOS}

\section{Sitios arqueológicos}

Trece individuos procedentes de cuatro sitios arqueológicos ubicados en dos regiones de la Provincia de Córdoba fueron estudiados: el sitio El Diquecito en las Llanuras, y los sitios El Vado, Guasmara y Loteo 5 en las Sierras Centrales (Fig. 1, Tabla 1). El Diquecito se encuentra en la costa sudoeste de la Laguna Mar Chiquita, en el noreste de la provincia. Es un sitio al aire libre altamente visible, de aproximadamente $500 \mathrm{~m}$ de largo por 500 de ancho. Once individuos juveniles y un adulto fueron recuperados, de los cuales sólo nueve contenían sedimentos abdominales, fechados mediante AMS entre $2562 \pm 47$ y $500 \pm$ $57{ }^{14} \mathrm{C}$ años AP (Fabra, González y Salega, 2012). El Vado es un sitio a cielo abierto ubicado en el Departamento Punilla, en el cañón del río Santa Catalina, donde aparecieron restos humanos pertenecientes a un único individuo, datado por AMS en $2156 \pm 86{ }^{14} \mathrm{C}$ años BP (Fabra y Demarchi, 2013). Loteo 5 también es un sitio a cielo abierto, excavado en 2011. Se encuentra ubicado en una propiedad privada en la ciudad de Santa Rosa de Calamuchita, donde se halló un entierro primario doble, con cerámicas, cáscaras de huevos, y caracoles asociados. El individuo 1 fue datado en $533 \pm 42{ }^{14} \mathrm{C}$ años AP (Fabra y González, 2019). Finalmente, el sitio Guasmara fue excavado en 1998. Se encuentra ubicado en la localidad Villa de las Rosas, Departamento San Javier, en una barranca dentro de un campo privado. A pesar de que se hallaron restos pertenecientes a dos individuos, sólo uno contenía sedimentos abdominales (individuo 1). El individuo 2 fue fechado por AMS en $920 \pm 20{ }^{14} \mathrm{C}$ años AP (Laguens et al., 2009) (Fig. 2).

Se analizaron muestras de sedimentos $(n=20)$, principalmente de la cavidad pélvica de los individuos, pero también de la cavidad abdominal y la superficie del sacro, tomados durante el trabajo de campo. Sedimentos de los forámenes del sacro, extraídos en el laboratorio después de la limpieza de los restos, también fueron procesados (Tabla 1). Además, se analizaron muestras control del sitio, consistentes en sedimentos del cráneo de los individuos, para descartar una posible contaminación ambiental. 


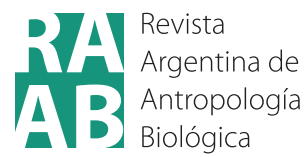

Biológica

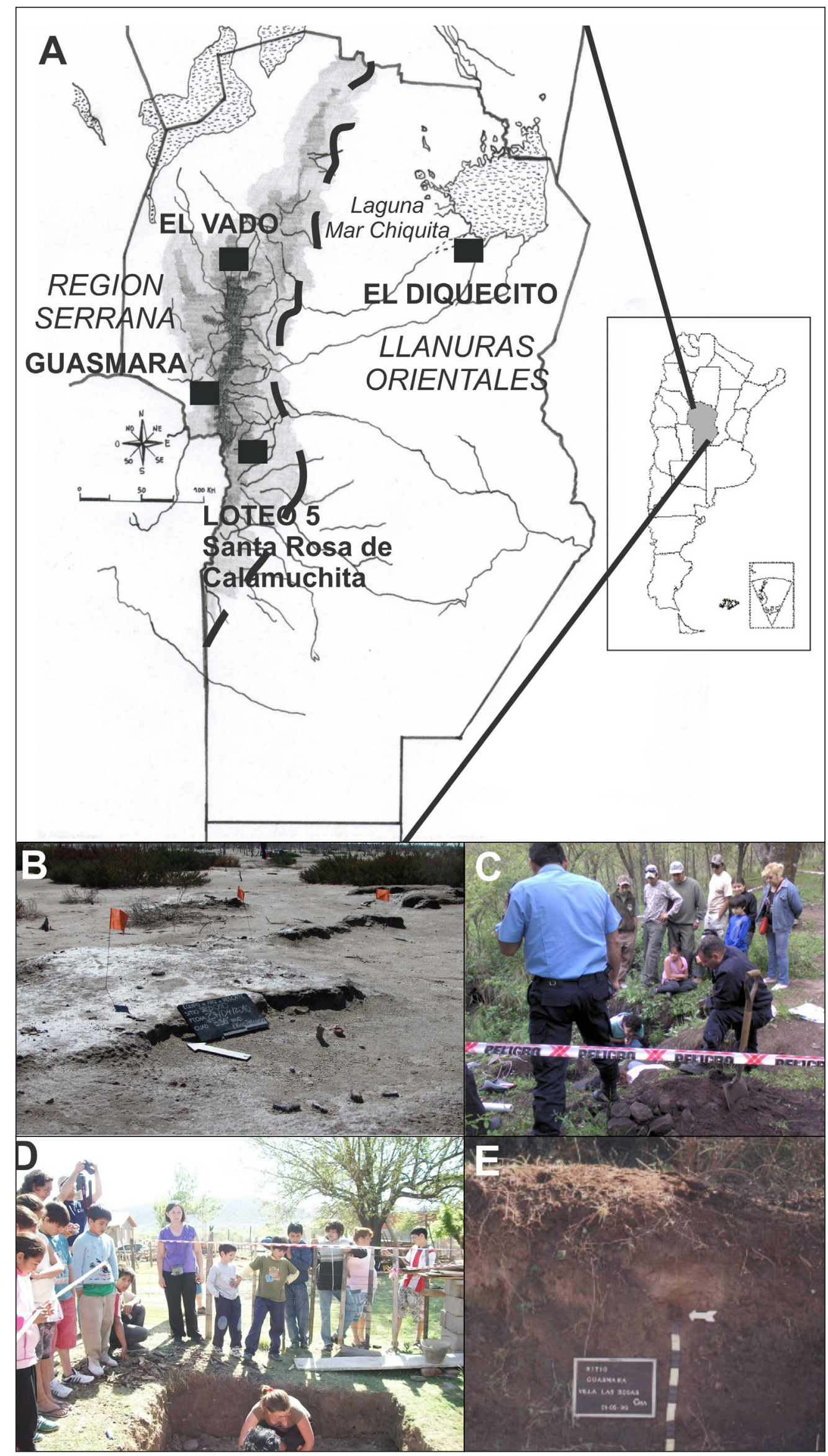

FIGURA 1. A) Mapa de la Provincia de Córdoba mostrando la ubicación de los sitios arqueológicos de proveniencia de las muestras estudiadas; B) Sitio El Diquecito; C) Sitio El Vado; D) Sitio Loteo 5; E) Sitio Guasmara. 
TABLA 1. Información bioarqueológica de los individuos y origen de las muestras analizadas

\begin{tabular}{|c|c|c|c|c|c|c|}
\hline Región & $\begin{array}{l}\text { Sitio } \\
\text { Arqueológico }\end{array}$ & Individuo & Sexo & Edad & $\begin{array}{l}\text { Datación radiocarbónica } \\
\text { (código de fechado) }\end{array}$ & $\begin{array}{c}\text { Origen } \\
\text { de las } \\
\text { muestras }\end{array}$ \\
\hline \multirow[t]{15}{*}{ Llanuras } & El Diquecito & CRI1 & $\mathrm{F}$ & $42-87$ & 2562 \pm 47 (AA93742) & $\mathrm{CP}$ \\
\hline & & CQI1 & M & 45 & 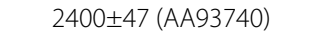 & FS \\
\hline & & & & & & $C P$ \\
\hline & & CSI1 & M & $33-42$ & $2331 \pm 46($ AA93745) & FS \\
\hline & & CGI1 & $\mathrm{F}$ & $33-46$ & 1872士59 (MTC14023) & FS \\
\hline & & & & & & $\mathrm{CP}$ \\
\hline & & CEl1 & M & $42-49$ & 1192 140 (MTC13247) & FS \\
\hline & & & & & & $\mathrm{CP}$ \\
\hline & & CLI1 & M & $33-42$ & 937士150 (MTC13214) & $\mathrm{CP}$ \\
\hline & & CBI1 & M & $20-23$ & 750 185 (MTC12807) & FS \\
\hline & & & & & & $C P$ \\
\hline & & CAl1 & F & $33-46$ & $698 \pm 42$ (AA93743) & FS \\
\hline & & & & & & $C P$ \\
\hline & & CMI1 & $\mathrm{F}$ & $42-87$ & $500 \pm 57(\mathrm{MTC} 14022)$ & FS \\
\hline & & & & & & $\mathrm{CP}$ \\
\hline \multirow{4}{*}{$\begin{array}{l}\text { Sierras } \\
\text { Centrales }\end{array}$} & El Vado & EVI1 & $\mathrm{F}$ & $30-44$ & 2156士86 (MTC12808) & FS \\
\hline & Guasmara & Gl1 & M & $20-24$ & $\begin{array}{c}920 \pm 20 \text { (UCI 22281) (Por } \\
\text { asociación con Individuo 2) }\end{array}$ & FS \\
\hline & Loteo 5 & L5I1 & $\mathrm{F}$ & $35-50$ & $533 \pm 42(\mathrm{AA} 102659)$ & FS \\
\hline & & $\mathrm{L} 512$ & $\mathrm{~F}$ & $43-71$ & $\begin{array}{c}533 \pm 42 \text { (AA102659) (Por } \\
\text { asociación con Individuo 1) }\end{array}$ & $\mathrm{S}-\mathrm{CA}$ \\
\hline
\end{tabular}

Individuo: CRI1, sitio El Diquecito, cuadrícula R, individuo 1; CQI1: Sitio El Diquecito, Cuadrícula Q, individuo 1; CSI1: sitio El Diquecito, cuadrícula S, individuo 1; CGI1: sitio El Diquecito, cuadrícula G, individuo 1; CEL1: sitio El Diquecito, cuadrícula E individuo 1 ; CLI1: sitio El Diquecito, cuadrícula L, individuo 1; CBI1: sitio El Diquecito, cuadrícula B, individuo 1; CAl1: sitio El Diquecito, cuadrícula A, individuo 1; CMI1: sitiio El Diquecito, cuadrícula M individuo 1; EVI1: sitio El Vado individuo 1: GI1: sitio Guasmara individuo 1; L5I1: sitio Loteo 5 Santa Rosa de Calamuchita, individuo 1; L5I2: sitio Loteo 5 Santa Rosa de Calamuchita, individuo 2; Sexo: F: Femenino, M: Masculino, Laboratorio donde se realizó el fechado radiocarbonico: AA: Universidad de Arizona, MTC: Universidad de Tokio, UCl: Universidad de Irving; Origen de las muestras: cavidad pélvica, FS: forámenes del sacro, CA: cavidad abdominal, S: sacro.

\section{Metodología}

Se rehidrataron $5 \mathrm{~g}$ de sedimento de cada muestra, incluidos los controles en una solución de fosfato trisódico al 0,5\% (Callen y Cameron, 1960) durante $72 \mathrm{~h}$ a $4^{\circ} \mathrm{C}$. Con el objetivo de obtener la mayor cantidad de hallazgos posibles, se aplicaron tres técnicas de concentración basadas en distintos principios: sedimentación espontánea (Lutz, 1919), disgregación en ácido clorhídrico al 10\% (Reinhard, Araújo, Sianto, Costell y Swope, 2008 con modificaciones) y flotación en solución de sacarosa $(\delta=1290)$ (Sheather, 1923). Se separaron alícuotas de $200 \mu \mathrm{L}$ con las dos primeras técnicas, mientras que se observó un único preparado con la flotación en sacarosa después de media hora. Los preparados fueron observados con un microscopio óptico trinocular (Labklass XSZ 107 CCD) con 100X (aumentos totales), y los restos parasitarios fueron fotografiados con 400X y medidos con el software IS Capture versión 3.5.9. El diagnóstico de los parásitos fue hecho en base a sus características morfológicas y morfométricas (Atías, 1998; Rey, 2008).

\section{RESULTADOS}

Se detectó la presencia de estructuras sugestivas de huevos o larvas de nemátodos en los cuatro sitios arqueológicos estudiados durante los análisis paleoparasitológicos (Tabla 2). En general, los restos se encontraron con deformaciones, daños o con detritus adherido, lo cual obstaculizó el diagnóstico diferencial. Los restos fueron recuperados mediante las técnicas de sedimentación espontánea y disgregación en ácido clorhídrico. La flotación en 


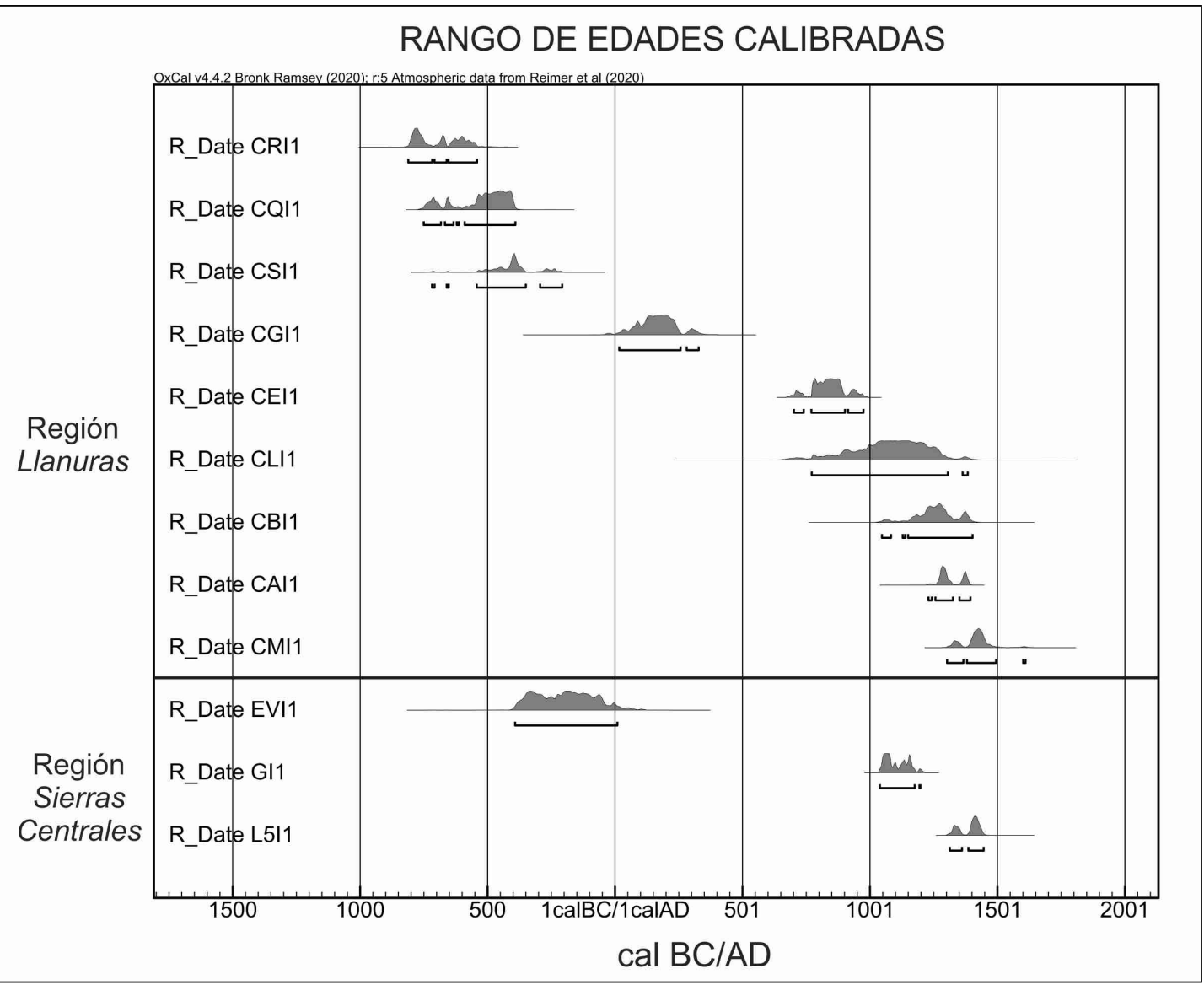

FIGURA 2. Fechados radiocarbónicos calibrados para los individuos analizados en el presente trabajo.

TABLA 2. Resultados del análisis paleoparasitológico en las muestras estudiadas

\begin{tabular}{|c|c|c|c|c|}
\hline \multirow[t]{2}{*}{ Sitio } & \multirow[t]{2}{*}{ Individuo } & \multirow[t]{2}{*}{ Resultado Paleoparasitológico } & \multicolumn{2}{|c|}{ Medidas de Estructuras Parasitarias } \\
\hline & & & Largo & Ancho \\
\hline \multirow[t]{9}{*}{ El Diquecito } & CRI1 & Negativo & - & - \\
\hline & CQI1 & Negativo & - & - \\
\hline & CSI1 & Negativo & - & - \\
\hline & CGI1 & Negativo & - & - \\
\hline & CEl1 & Negativo & - & - \\
\hline & CLI1 & Negativo & - & - \\
\hline & CBI1 & Negativo & - & - \\
\hline & CAl1 & Huevo de Heterakoidea & $66 \mu \mathrm{m}$ & $57 \mu \mathrm{m}$ \\
\hline & CMI1 & Huevo de Heterakoidea & $77,33 \mu \mathrm{m}$ & $61,81 \mu \mathrm{m}$ \\
\hline El Vado & EVI1 & Sugestivo de huevo de Heterakoidea & $44,89 \mu \mathrm{m}$ & $48,17 \mu \mathrm{m}$ \\
\hline \multirow[t]{3}{*}{ Guasmara } & Gl1 & Larva de nematodo NI & $560,76 \mu \mathrm{m}$ & $18,17 \mu \mathrm{m}$ \\
\hline & & Sugestivo de huevo de nematodo $\mathrm{NI}$ & $51,31 \mu \mathrm{m}$ & $36,81 \mu \mathrm{m}$ \\
\hline & & Sugestivo de huevo de Ascaridoidea & $65,29 \mu \mathrm{m}$ & $40,10 \mu \mathrm{m}$ \\
\hline \multirow[t]{2}{*}{ Loteo 5} & L5I1 & Larva de nematodo NI & - & - \\
\hline & $\llcorner 512$ & Huevo de nematodo $\mathrm{NI}$ & $55,88 \mu \mathrm{m}$ & $44,17 \mu \mathrm{m}$ \\
\hline
\end{tabular}

Individuo: CRI1, sitio El Diquecito, cuadrícula R, individuo 1; CQI1: Sitio El Diquecito, Cuadrícula Q, individuo 1; CSI1: sitio El Diquecito, cuadrícula S, individuo 1; CGI1: sitio El Diquecito, cuadrícula G, individuo 1; CEL1: sitio El Diquecito, cuadrícula E individuo 1; CLI1: sitio El Diquecito, cuadrícula L, individuo 1; CBI1: sitio El Diquecito, cuadrícula B, individuo 1; CAl1: sitio El Diquecito, cuadrícula $\mathrm{A}$, individuo 1; CMI1: sitiio El Diquecito, cuadrícula M individuo 1; EVI1: sitio El Vado individuo 1: GI1: sitio Guasmara individuo 1; L5I1: sitio Loteo 5 Santa Rosa de Calamuchita, individuo 1; L5I2: sitio Loteo 5 Santa Rosa de Calamuchita, individuo 2: Resultado paleoparasitologico: NI: No identificado; Unidad de medida: $\mu \mathrm{m}$ : micrón. 
solución de sacarosa no evidenció resultados positivos. En todos los casos, se hallaron escasos restos parasitarios, que no pudieron ser identificados al nivel taxonómico de género o especie. Al contrario, una gran cantidad de esporas de hongos, granos de polen y otros microrestos no identificados fueron observados.

En el individuo del sitio Guasmara, una estructura similar a un huevo de nematodo no identificado ( $51.31 \mu \mathrm{m} \times 36.81 \mu \mathrm{m})$ (Fig. 3A) con pared doble y parcialmente dañada fue observada. Además, se halló una estructura que sugiere un huevo de Ascaridoidea $(65.29 \mu \mathrm{m} x$ $40.10 \mu \mathrm{m})$, de color pardo, forma elongada y cobertura mamelonada, típica de los ascarídi-

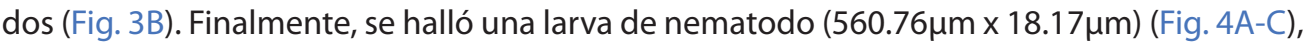
posiblemente de vida libre, por lo que no consideramos a este hallazgo como un resultado positivo de parasitosis.

En el individuo estudiado del sitio El Vado fue identificado un huevo sugestivo de Heterakoidea, que midió $44.89 \mu \mathrm{m}$ x 48.17 $\mu \mathrm{m}$ (Fig. 3C). Un individuo del sitio Loteo 5 (L5I2) evidenció la presencia de una estructura de huevo de nematodo no identificado con una pared doble y medidas de $55.88 \mu \mathrm{m} \times 44.17 \mu \mathrm{m}$ (Fig. 3D). En el otro individuo analizado de este sitio (L5I1) se observó una larva de helminto no identificada, la cual se encontraba enroscada, impidiendo su medición (Fig. 4D). Todas las muestras control arrojaron resultados negativos para estos sitios.

Uno de los nueve individuos (CMI1) estudiados del sitio El Diquecito reveló la presencia de un huevo de Heterakoidea $(73.33 \mu \mathrm{m}$ x $81.81 \mu \mathrm{m})$ con forma ligeramente ovoide y contenido amorfo (Fig. 3E). La muestra control no evidenció contaminación ambiental. En el individuo CAI1, donde no se hallaron restos parasitarios en la cavidad pélvica, se identificó un huevo de Heterakoidea de doble pared $(66 \mu \mathrm{m} \times 57 \mu \mathrm{m})$ en la muestra control tomada del cráneo (Fig. 3F).

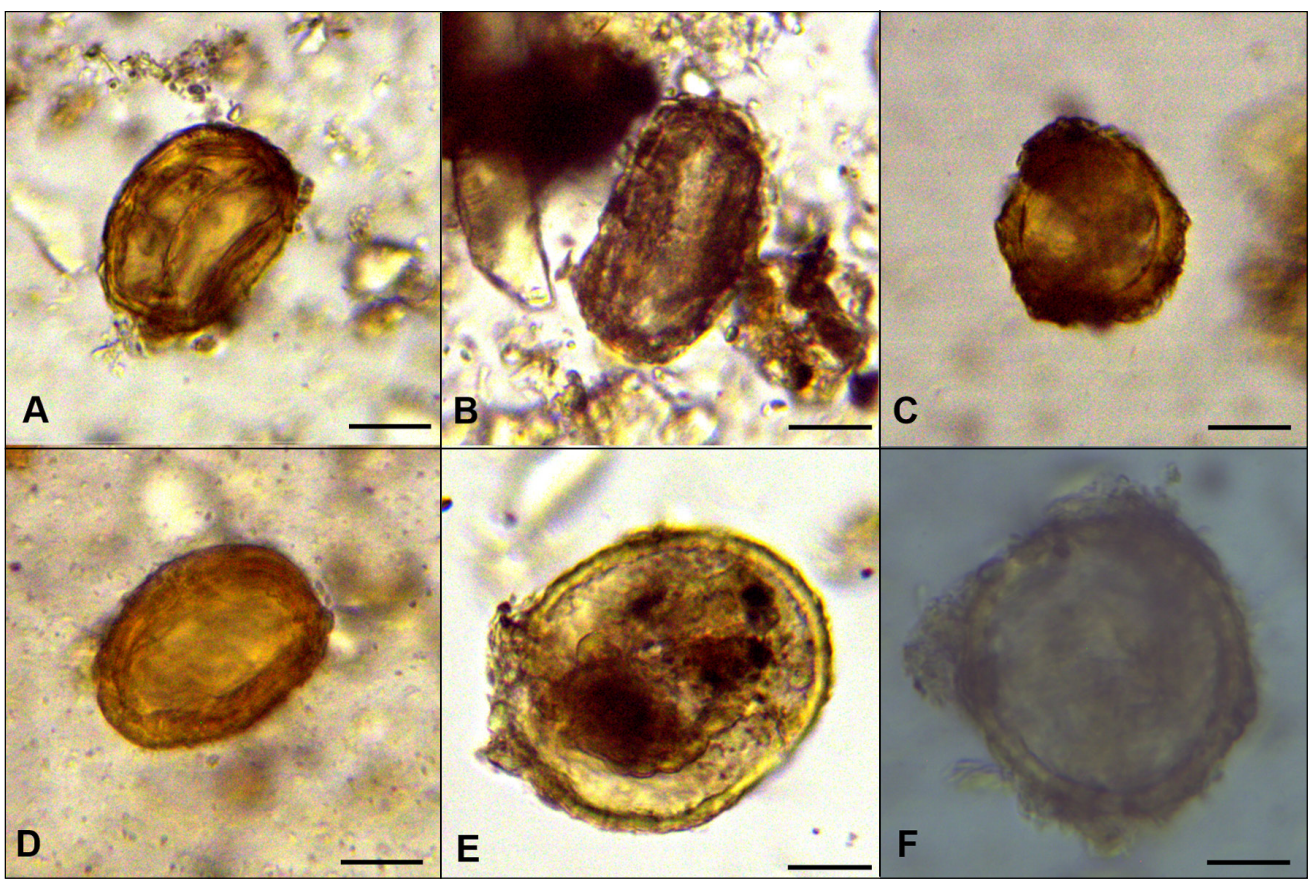

FIGURA 3. Resultados del análisis paleoparasitológico. A) Estructura similar a un huevo de nematodo no identificado; B) Estructura sugestiva de huevo de Ascaridoidea; C) Estructura sugestiva de huevo de Heterakoidea; D) Huevo de nematodo no identificado; E) Huevo de Heterakoidea; F) Huevo de Heterakoidea. Escala $=20 \mu \mathrm{m}$. 


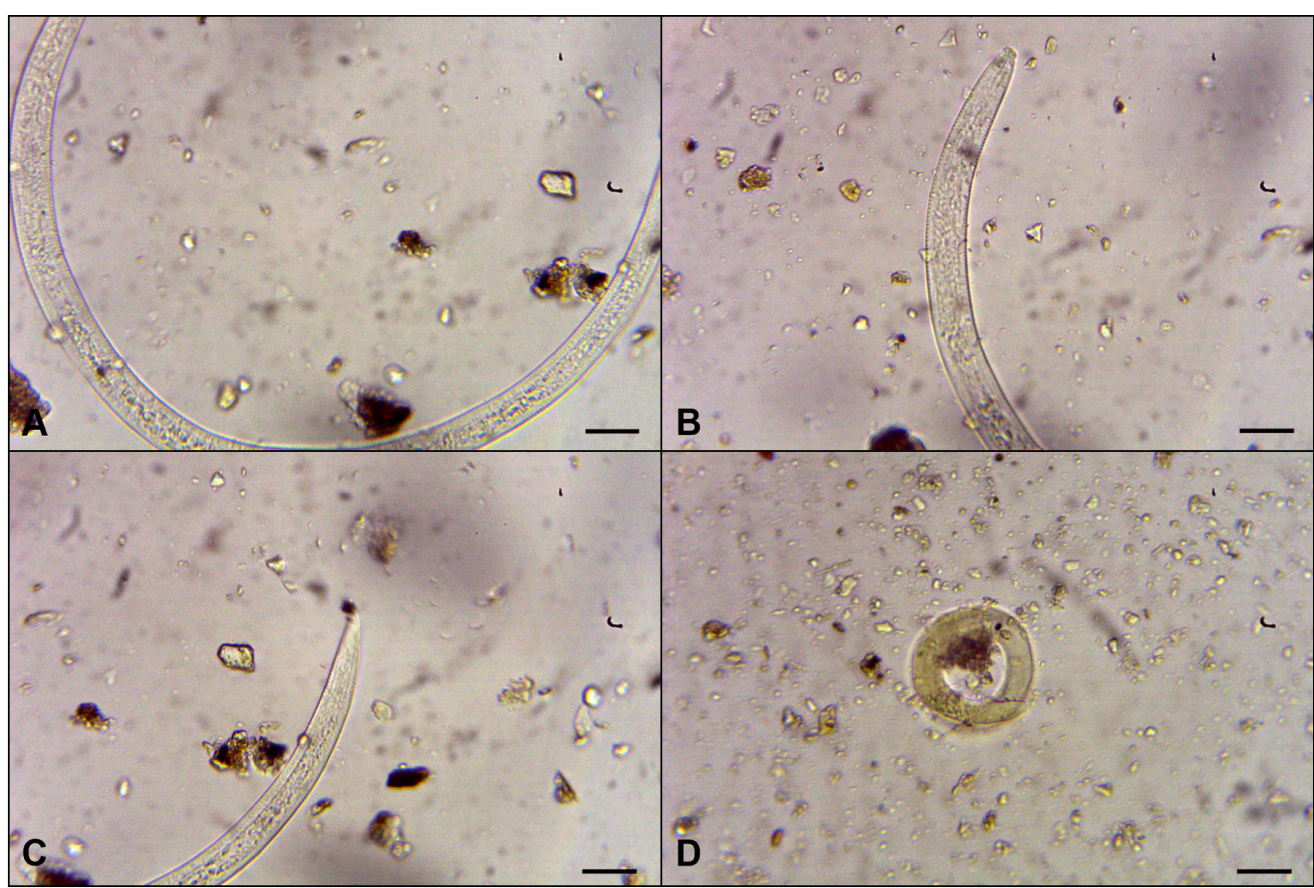

FIGURA 4. Resultados del análisis paleoparasitológico. A-C) Larva hallada en el individuo del sitio Guasmara (A. Porción media; B. Porción anterior; C. Porción posterior). D) Larva hallada en el individuo 1 del sitio Loteo 5. Escala $=20 \mu \mathrm{m}$.

\section{DISCUSIÓN Y CONCLUSIONES}

El hallazgo de estructuras parasitarias en el presente trabajo confirma que las poblaciones que vivieron en las diferentes regiones de la Provincia de Córdoba, principalmente en las sierras, estuvieron expuestas a parásitos en el pasado. Desde una perspectiva ecológica, el parasitismo se presenta como un fenómeno natural complejo compuesto por parásito-huésped-ambiente, un sistema donde cada elemento interactúa e influye necesariamente con el otro (Ferreira, 1973). Las parasitosis pueden incluir casos de parasitismo verdadero o parasitismo falso o accidental, así como también de zoonosis parasitaria. En los eventos de falso parasitismo, el ser humano no es el huésped definitivo del parásito, por lo que no puede completar su ciclo en su organismo y es eliminado en sus heces. Generalmente, los casos de falso parasitismo en humanos se producen por la ingesta de partes de animales que son los huéspedes definitivos del parásito. Estos eventos han sido reportados como el resultado de la presencia de parásitos animales en restos humanos provenientes de sitios arqueológicos de todo el mundo (Le Bailly, 2005; Reinhard, 1990; Sianto et al., 2009; Vieira et al., 2018). Respecto a los casos de zoonosis parasitarias, los parásitos animales tienen éxito en establecerse en el cuerpo humano, completando parcial o totalmente su ciclo, causando manifestaciones clínicas (Sianto et al., 2009). Todos estos eventos pueden ser entendidos como interacciones ecológicas y la Paleoparasitología tiene el potencial de identificar estas interacciones en escenarios temporales.

Los parásitos hallados en este trabajo parasitan a un amplio espectro de animales, incluyendo aves y roedores (Chabaud, 1974; Hartwich, 1974; Kijewska, Rokicki, Sitko y Wegrzyn, 2002), pero no han sido reconocidos como agentes causales de parasitosis verdadera en humanos. En principio, con el hallazgo en el individuo del sitio Guasmara de una estructura sugestiva de un huevo de Ascaridoidea, superfamilia que incluye Ascaris sp., parásito del ser humano, no se podría descartar una genuina infección humana. 
Sin embargo, por la poca especificidad taxonómica se considera a este hallazgo como posible ascarídido de otros animales. Así, se sugiere que eventos de falso parasitismo podrían haber tenido lugar en estas poblaciones humanas. El registro zooarqueológico confirma la presencia de roedores y aves en algunos sitios arqueológicos de la provincia. Particularmente para las Sierras Centrales durante el Holoceno tardío, se ha propuesto la sustitución de la caza de grandes presas como el guanaco (Lama guanicoe), y los cérvidos como el ciervo de las pampas (Ozotoceros bezoarticus), por pequeños animales, incluyendo a los roedores cavidos (Caviidae) y los tucutucu (Ctemoys sp.), iguanas (Tupinambis sp.), "tinamúes" o mal llamados "perdices" (Tinamidae) (Rivero, Medina, Recalde y Pastor, 2010), y también un incremento en el consumo de aves de la familia Rheidae, patos (Anatidae), "vizcacha de las pampas" (Lagidium viscacia) y armadillos (Euphractinae) (Rivero y Medina, 2016). Puntualmente, se sugirió que la intensificación en la frecuencia de pequeños roedores en los conjuntos faunísticos desde el Holoceno Medio hasta el Tardío, fue consecuencia de un ajuste comportamental en respuesta a la disminución de L. guanicoe, debido a la sobre-explotación del ambiente por parte del humano. Además, los análisis isotópicos de $\delta^{13} \mathrm{C}_{\mathrm{col}}$ y ${ }^{815} \mathrm{~N}$ en algunos individuos estudiados en este trabajo, muestran valores coincidentes con una posible ingesta de aves y roedores por estas poblaciones (Fabra y González, 2019). En suma, los datos aportados por estudios zooarqueológicos e isotópicos nos permiten sugerir una dieta basada en estos animales como una forma de contacto entre parásitos enzoóticos y humanos. Otra explicación posible está relacionada con la ingesta de alimentos contaminados con heces de animales que cohabitaron el mismo espacio que los humanos. En ambos casos, la ocurrencia de los nematodos hallados en estos individuos estaría relacionada a una mayor proximidad con los animales, huéspedes intermedios o definitivos de estos parásitos, durante el Holoceno Tardío.

Se ha reportado el hallazgo de huevos de ascarídidos en materiales antiguos, tanto de origen arqueológico en América (Araújo et al., 2015; Fugassa, 2006; Fugassa y Barbarena, 2006), como paleontológico en sitios de Europa y Sudamérica, extendiéndose su registro hasta 100 y 240 millones de años, respectivamente (Da Silva et al., 2014; Poinar y Boucot, 2006). Por otro lado, la extensión temporal de los reportes de Heterakoidea en el pasado es mucho más reciente, restringida a un basurero arqueológico en Francia (Bouchet, 1991), dos sambaquis brasileños (Camacho et al., 2013, Camacho, Borba Cruz, Silva, Mendonça de Souza y Araújo, 2015) y coprolitos de megafauna extinta del Holoceno en Nueva Zelanda (Wood et al., 2013). La identificación de huevos sugestivos de Heterakoidea en individuos de los sitios EI Vado y El Diquecito es interesante, ya que sería el primer reporte paleoparasitológico de este parásito asociado directamente a los contenidos intestinales de restos humanos. La presencia de un huevo de Heterakoidea en sedimentos del cráneo del individuo CAl1 del sitio El Diquecito sugiere contaminación ambiental en este individuo, aunque no se hallaron huevos en su cavidad pélvica. La ocurrencia de estos parásitos en este individuo estaría relacionada con la presencia de animales, principalmente aves, en el sitio. Los huevos de esta Superfamilia tienen cáscara gruesa con granulación, difícil de distinguir entre las especies (Chabaud, 1978; Yevstafyeva et al., 2018), y pueden presentar cambios en sus bordes y estructura cuando se liberan al ambiente (Yevstafyeva et al., 2018). Además, la pobre preservación de material arqueológico puede resultar en la alteración de los huevos. Estos son factores limitantes para el diagnóstico preciso de las especies Heterakoidea.

Respecto al bajo número de restos hallados, consideramos que la composición de ciertos tipos de suelos puede ser un factor negativo para la preservación de parásitos. Un trabajo anterior (Ramirez, Xavier, Fabra e Iñiguez, 2017) evaluó la preservación de res- 


\section{AGRADECIMIENTOS}

A los organismos e instituciones que financiaron o facilitaron con sus instalaciones la realización de esta investigación. Al Dr. Rodrigo Nores (IDACOR-CONICET-UNC) por su ayuda constante durante el trabajo de laboratorio. tos parasitarios en suelos locales a partir de la elaboración de coprolitos experimentales, es decir, materia fecal con resultados parasitológicos positivos artificialmente desecados (Iñiguez, 1998), con añadidos de sedimentos de un sitio arqueológico de la provincia de Córdoba. Los resultados permitieron determinar la pérdida estadísticamente significativa de huevos de parásitos, especialmente en presencia del añadido de sedimento, cuando se comparó con muestras de materia fecal frescas, sin añadidos de suelo.

La escasez de parásitos hallados en El Diquecito posiblemente se debe también a la ubicación del sitio, parcialmente inmerso en la costa de una laguna, donde la temperatura y la humedad podrían haber permitido una importante biodegradación de los restos parasitarios. Además, los flujos y corrientes del agua generan percolación, es decir la migración de los restos hacia capas estratigráficas inferiores, un factor que se incrementa en sitios a cielo abierto (Fugassa, 2014).

Finalmente, el bajo número de estructuras halladas en todos los sitios arqueológicos difiere de los resultados para otras regiones del país, especialmente en Patagonia. Estos dos ambientes son extremadamente diferentes en términos de temperaturas, humedad y regímenes fluviales, los cuales influyen significativamente en la preservación de restos orgánicos y en la formación de coprolitos. Algunos ambientes, especialmente aquellos secos y áridos, sean fríos o cálidos, permiten una buena preservación general de muchos materiales estudiados comúnmente en Paleoparasitología, incluyendo cuerpos momificados y tejidos blandos, textiles, pellets de regurgitación y coprolitos (Ferreira, 2014). Ambientes húmedos, generalmente relacionados con regímenes fluviales intensos y alta actividad microbiana entre otros, no se caracterizan por dar lugar a una preservación ideal de estas materialidades. En la extensa región patagónica se encuentra una gran diversidad de ambientes, pero, en general, todos pueden ser descriptos como fríos y secos (Guichón, Trivi y Burry, 2014). Por el contrario, en la región central del país, los períodos de mayor humedad o sequía tienen ciclos estacionales, coincidiendo con los semestres más cálidos o más fríos del año, respectivamente (Furgioni, Salvatierra y Tuninetti, 2016). Además, estudios paleoambientales realizados sobre multi-indicadores (sedimentología, geoquímica, isótopos estables, restos biológicos) en la Laguna Mar Chiquita han permitido estimar la alternancia de periodos de mayor aridez y balance hídrico negativo, en contraposición con momentos de mayor humedad y niveles elevados de agua durante los últimos 20.000 años (Piovano, Zanor y Ariztegui, 2006), lo cual seguramente impactó no solo en la ocupacion humana efectiva de estos territorios, sino en las condiciones de preservación de los materiales arqueológicos. Debido a estas características ambientales, los coprolitos no se forman en la región central del país, dejando a los sedimentos, principalmente a los de la cavidad pélvica de esqueletos, como el único tipo de muestras para estudios paleoparasitológicos de parásitos intestinales. La diferencia en términos de preservación de las estructuras parasitarias entre sedimentos y coprolitos es importante, ya que estos últimos brindan, además de una mejor preservación, mayores posibilidades de identificación de ciertos parásitos a niveles taxonómicos precisos (Borba, Machado-Silva, Le Bailly e Iñiguez, 2019). Así, la pérdida de parásitos en las muestras analizadas en el presente trabajo podría deberse a la acción de efectos tafonómicos, tales como el calor de la desecación y la composición orgánica e inorgánica del suelo. Sin embargo, no puede descartarse que las parasitosis tuvieran una baja carga parasitaria en las poblaciones que habitaron el actual territorio de la provincia de Córdoba en el Holoceno Tardío.

En resumen, los estudios paleoparasitológicos presentados en este trabajo sugieren que las poblaciones que habitaron esta región estuvieron expuestas a parásitos, posiblemente de origen animal, al menos desde hace 2100 años AP. Los hallazgos se consideran 
evidencias de interacciones culturales y ecológicas entre humanos y animales, que dieron como resultado eventos de falso parasitismo. Este primer estudio paleoparasitológico en la región contribuye al conocimiento de los modos de vida de estas poblaciones en el Holoceno Tardío. La aplicación de técnicas más sensibles, como las empleadas en Paleoparasitología Molecular, será abordada a futuro, con el objetivo de alcanzar una identificación más precisa de los restos hallados, incluso en muestras con resultados negativos mediante microscopía (Iñiguez et al., 2006; Iñiguez, 2014).

\section{LITERATURA CITADA}

Araújo, A, Ferreira, L.F., Fugassa, M.H., Leles, D., Sianto, L., Mendonça de Souza, ... Reinhard, J.K. (2015). New World Paleoparasitology. En Mitchell, P. (Ed) Sanitation, Latrines and Intestinal Parasites in Past Populations (pp. 165-186). London, England: Ashgate.

Araújo, A., Reinhard, J. K., Ferreira, L. F., Pucu, E. y Chieffie, P. P. (2013). Paleoparasitology: the origen of human parasites. Arquivos de Neuropsiquiatria, 71, 722-726. doi:10.1590/0004282X20130159

Araujo, A., Reinhard, J. K., Ferreira, L. F. y Gardner, S. (2008). Parasites as probes for prehistoric human migrations? Trends in Parasitology, 24(3), 112-115. doi:10.1016/j.pt.2007.11.007

Atías, A. (1998). Parasitología médica. Santiago, Chile: Editorial Mediterráneo.

Beltrame, M. O., Vieira de Souza, M., Araújo, A. y Sardella, N. H. (2014). Review of the rodent paleoparasitological knowledge from South America. Quaternary International, 352, 68-74. doi:10.1016/j. quaint.2014.09.006.

Beltrame, M. O., Pruzzo, C., Sanabria, R., Pérez, A. y Mora, M. S. (2020). First report of pre-Hispanic Fasciola hepatica from South America revealed by ancient DNA. Parasitology, 147(3), 371-375. doi:10.1017/S0031182019001719.

Borba, V. H., Machado-Silva, J. R., Le Bailly, M. e Iñiguez A. M. (2019). Worldwide paleodistribution of capillariid parasites: Paleoparasitology, current status of phylogeny and taxonomic perspectives. PLoS ONE, 14(4), e0216150. doi:10.1371/journal.pone.0216150

Bouchet, F. (1991). Étude parasitologique des chantiers archéologiques de Beauvais (Oise), Revue Archéologique de Picardie, 3/4, 293-294. doi:10.3406/pica.1991.1636

Callen, E. O. y Cameron, T.W.M. (1960). A prehistoric diet revealed in coprolites. New Scientist, 8, 35-40.

Camacho, M., Pessanha, T., Leles, D., Dutra, J.M.F., Rosângela, S., Mendonça de Souza, S. y Araújo, A. (2013). Lutz's spontaneous sedimentation technique and the paleoparasitological analysis of sambaqui (shell mound) sediments. Memórias do Instituto Oswaldo Cruz, 108(2), 155-159.

Camacho, M., Borba Cruz, P., Silva, V. L., Mendonça de Souza, S. y Araújo, A. (2015). Uso da técnica de ácido clorídrico a $10 \%$ e de técnicas de imunodiagnóstico para recuperar vestígios de parasitos de amostras de sedimentos provenientes de um sambaqui. En Luna, L. H., Aranda, C. M., Suby, J. A., Guichón, R. A., Flensborg, G. A. y García Laborde, P. (Eds.) Libro de Resúmenes VI Paleopathology Association Meeting in South America (pp 121-123). Buenos Aires, Argentina: Editorial GIB.

Chabaud, A. (1974). Keys to Genera of The Superfamilies Cosmocercoidea, Seuratoidea, Heterakoidea and Subuluroidea. En Anderson, R., Chabaud, A. y Willmott, S. (Eds.) ClH Keys to the Nematode Parasites of Vertebrates. Slough, England: Commonwealth Agricultural Bureaux.

Da Silva, P. A., Borba, V. H., Dutra, J. M. F., Leles, D., Da-Rosa, A. A. S., Ferreira, L. F. y Araújo, A. (2014). A new ascarid species in cynodont coprolite dated of 240 million years. Anais da Academia Brasileira de Ciências, 86(1), 265-269. doi:10.1590/00013765201320130036

Fabra, M. y Demarchi, D. A. (2013). Análisis morfogeométrico aplicado al estudio de los patrones espaciales y temporales de variación morfológica craneofacial en poblaciones del centro de Argentina. Cuadernos del Instituto Nacional de Pensamiento Latinoamericano, 1(1), 87-101.

Fabra, M. y González, C. (2019). Oral health and diet in populations of Central Argentina during late 
Holocene: bioarchaeological and isotopic evidence. Latin American Antiquity, 30(4), 818-835. doi:10.1017/laq.2019.69

Fabra, M., González, C. V. y Salega M. S. (2012). Modos de vida e historia biológica de poblaciones de las Sierra y Llanuras de Córdoba (Argentina): aproximaciones desde el registro bioarqueológico. Revista Argentina de Antropología Biológica, 14, 87-104.

Ferreira, L. F. (1973). O fenómeno parasitismo. Revista da Sociedade Brasileira de Medicina Tropical, 7 (4): 261-277. doi:10.1590/S0037-86821973000400006

Ferreira, L. F. (2014). The Preservation of Organic Material over Time. En Ferreira, L. F., Reinhard, J. K. y Araújo, A (Eds.) Foundations of Paleoparasitology (pp 43-52). Río de Janeiro, Brasil: Editora Fiocruz.

Fugassa, M. H. (2006). Examen paleoparasitológico de sedimentos de un sitio arqueológico, Río Mayo, Chubut, Argentina. Parasitología Latinoamericana, 61, 172-175.

Fugassa, M. H. (2014). Paleoparasitological Diagnosis. En Ferreira, L.F., Reinhard, J.K. y Araújo, A (Eds.) Foundations of Paleoparasitology (pp. 223-254). Río de Janeiro, Brasil: Editora Fiocruz.

Fugassa, M. H. y Barberena, R. (2006). Cuevas y zoonosis antiguas: paleoparasitología del Sitio Orejas de Burro 1 (Santa Cruz, Argentina). Magallania, 34(2), 57-62.

Fugassa, M. H., Beltrame, M. O., Sardella, N. H., Civalero, M. T. y Aschero, C. (2010). Paleoparasitological results from coprolites dated at the Pleistocene-Holocene transition as source of paleoecological evidences in Patagonia. Journal of Archaeological Science, 37, 880-884. doi:10.1016/j. jas.2009.11.018

Fugassa, M. H., Petrigh, R. S., Fernández, P. M., Carballido Catalayud, M. y Belleli, C. (2018). Fox parasites in Pre-columbian times: Evidence from the past to understand the current helminth assemblages. Acta Tropica, 185, 380-384. doi:10.1016/j.actatropica.2018.06.007

Fugassa, M. H. y Petrigh, R. S. (2019). Primeros registros de helmintos en roedores del sitio arqueológico Cueva Milodón Norte 1. Cuadernos del Instituto Nacional de Antropología y Pensamiento Latinoamericano, 28(1), 57-63.

Fugassa, M. H., Sardella, N. H., Guichón, R. A., Denegri, G. M. y Araújo, A. (2008). Paleoparasitological analysis applied to museum-curated sacra from Meridional Patagonian collections. Journal of Archaeological Science, 35, 1408-1411. doi:10.1016/j.jas.2007.10.006

Furgioni F., Salvatierra P. y Tuninetti. L. (2016). Estudio de las variaciones temporales del clima en la Región Central de la Provincia de Córdoba. II Jornadas de Desarrollo Local Regional. Reflexiones y diálogos para la acción. Universidad Nacional de Villa María. Villa María, Argentina.

Guichón R. A., Trivi M. y Burry L. (2014). Human occupation of Patagonia. En: Ferreira L.F., Reinhard J.K. y Araújo A. Foundations of Paleoparasitology (pp. 93-110). Editora Fiocruz. Río de Janeiro, Brasil.

Hartwich, G. (1974). Keys to genera of the Ascaridoidea. En Anderson, R., Chabaud, A. y Willmott, S. (Eds.) ClH Keys to the Nematode Parasites of Vertebrates. Slough, England: Commonwealth Agricultural Bureaux.

Iñiguez, A. M. (1998). Análise de DNA ancestral para o estudo de doenças parasitárias em populações pré-históricas. Tesis de Maestría. Instituto Oswaldo Cruz, Fundação Oswaldo Cruz. Río de Janeiro, Brasil.

Iñiguez, A. M., Reinhard, J. K., Gonçalves, M. L., Ferreira, L. F., Araújo, A. y Vicente, A. C. (2006). SL1 RNA gene recovery from Enterobius vermicularis ancient DNA in pre-Columbian human coprolites. International Journal for Parasitology, 36(13), 1419-1425.

Iñiguez, A. M. (2014). Paleoparasitologia Molecular e Paleogenética para o Estudo das Doenças Infecto-Parasitarias do Passado: Princípios, Metodologia e Agravos. En Luna L.H., Aranda C. M. y Suby J. A. (Eds.) Avances de la bioarqueología latinoamericana (pp 285-319). Ciudad Autónoma de Buenos Aires, Argentina: Grupo de Investigación en Bioarqueología GIB.

Kijewska, A., Rokicki, J., Sitko, J. y Wegrzyn, G. (2002). Ascaridoidea: a simple DNA assay for identification of 11 species infecting marine and freshwater fish, mammals and fish-eating birds. Experi- 
mental Parasitology, 101, 35-39.

Laguens, A. G., Fabra, M., Dos Santos, G. M. y Demarchi, D. A. (2009). Paleodietary inferences based on isotopic evidences for populations of the Central Mountains of Argentina during the Holocene. International Journal of Osteoarchaeology, 19(2), 237-249. doi:10.1002 /oa. 1064

Le Bailly, M., Gonçalves, M. L. C., Lefèvre, C., Roper, D. C., Pye, J.W., Araújo, A. y Bouchet, F. (2006). Parasitism in Kansas in the 1800s- a glimpse to the past through the analysis of grave sediments from Meadowlark cemetery. Memórias do Instituto Oswaldo Cruz, 101(Suppl. II), 53-56.

Le Bailly, M. (2005). Evolution de la relationhôte/parasite dans les systèmes lacustres nordalpins au Néolithique (3900-2900 BC), et nouvelles données dans la détection des paléoantigènes de Protozoa. Tesis de Doctorado. Universite de Reims Champagne-Ardenne, Francia.

Lutz, A. (1919). Schistosoma mansoni e a schistosomatose segundo observacoes feitas no Brasil. Memórias do Instituto Oswaldo Cruz, 11, 121-155.

Nores, R., Fabra, M., García, A. y Demarchi, D. A. (2017). Diversidad genética en restos humanos arqueológicos del sitio El Diquecito (Costa Sur, Laguna Mar Chiquita, Provincia de Córdoba). Revista Argentina de Antropología Biológica, 19, 7-18.

Piovano E. L., Zanor G. A. y Ariztegui D. (2006). Historia geológica y registro climático. En Bucher, E. H. (Ed.) Bañados del río Dulce y Laguna Mar Chiquita (Córdoba, Argentina) (pp. 37-55). Academia Nacional de Ciencias. Córdoba, Argentina.

Poinar, G. y Boucot, A. J. (2006). Evidence of intestinal parasites of dinosaurs. Parasitology, 133, 245249. doi:10.1017/S0031182006000138

Ramirez, D. A., Xavier, S., Fabra, M. e Iñiguez, A. M. (2017). Evaluation of the effect of desiccation and local soils in the recovery of parasites by experimental coprolites. VII Paleopathology Association Meeting in South America. Arica, Chile.

Reinhard, J. K. (1990). Archaeoparasitology in North America. American Journal of Physical Anthropology, 82, 145-163.

Reinhard, J. K., Araújo, A., Sianto, L., Costello, J. G. y Swope, K. (2008). Chinese Liver Flukes in latrine sediments from Wong Nim's Property, San Bernardino, California: Archaeoparasitology of the Caltrans District Headquarters. Journal of Parasitology, 94(1), 300-303. doi:10.1645/GE -1049.1

Rey, L. (2008). Parasitologia. 4th ed. Rio de Janeiro, Brasil: Guanabara Koogan.

Rivero, D., Medina, M., Recalde, A. y Pastor, S. (2010). Variabilidad en la explotación de recursos faunísticos durante el Holoceno en las Sierras de Córdoba (Argentina): una aproximación zooarqueológica. En Gutiérrez M., De Negris M., Fernández P., Giardina M., Gil A., Izeta A.D., Neme G. y Yacobaccio, H. (Eds.) Zooarqueología a principios del Siglo XXI: Aportes teóricos, metodológicos y casos de estudio (pp 321-332). Buenos Aires, Argentina: Ediciones El Espinillo.

Rivero, D. y Medina, M. (2016). Human Holocene colonization, dieth breath and niche construction in Sierras of Cordoba (Argentina). En Cardillo M. y Muscio, H. (Eds.) Darwin's Legacy: The Status of Evolutionary Archaeology in Argentina (pp 67-81). Oxford, England: Archaeopress Publishing Ltd.

Salega, S. y Fabra, M. (2013). Niveles de actividad física en poblaciones de las sierras y las Ilanuras de la provincial de Córdoba (Argentina) durante el Holoceno tardío. Relaciones de la Sociedad Argentina de Antropología, 38(2), 401-420.

Sheather, A. L. (1923). The detection of intestinal protozoa and mange parasites by a flotation technique. Journal of Comparative Pathology and Therapeutics, 36, 266-275. doi:10.1016/S0368$1742(23) 80052-2$

Sianto, L., Chame, M., Silva, C. S. P., Gonçalves, M. L. C., Reinhard, J.K., Fugassa, M. H. y Araújo, A. (2009). Animal helminthes in human archaeological remains: a review of zoonoses in the past. Revista do Instituto de Medicina tropical de Sao Paulo, 51(3), 119-130. doi:10.1590/S0036-46652009000300001

Tavarone, A., Colobig, M. y Fabra, M. (2019). Late Holocene plant use in lowland central Argentina: microfossil evidence from dental calculus. Journal of Archaeological Science: Reports 26. 101895. 
Tietze, E., Barberena, R. y Beltrame, M. O. (2019). Parasite Assemblages from Feline Coprolites through the Pleistocene-Holocene Transition in Patagonia: Cueva Huenul 1 Archaeological Site (Argentina). Environmental Archaeology, 1-11. doi:10.1080/14614103.2019.1689893.

Vieira de Souza, M., Ribeiro da Silva, L. G., Silva-Pinto, V., Mendez-Quiros, P., De Miranda Chaves, S. A. e Iñiguez, A. M. (2018). New paleoparasitological investigations from the pre-inca to hispanic contact period in northern Chile. Acta Tropica, 178, 290-296. doi:10.1016/j.actatropica.2017.11.021

Wood, J. R., Wilmshurst, J. M., Rawlence, N. J., Bonner, K. I., Worthy, T. H., Kinsella, J. M. y Cooper, A. (2013). A megafauna's microfauna: Gastrointestinal parasites of New Zealand's extinct moa (Aves: Dinornithiformes). PLoS One, 8(2), 1-9. doi:10.1371/journal.pone.0057315

Yeh, H., Prag, K., Clamer, C., Humbert, J. y Mitchell, P. (2015). Human intestinal parasites from a Mamluk Period cesspool in the Christian quarter of Jerusalem: Potential indicators of long distance travel in the 15th century AD. International Journal of Paleopathology, 9, 69-75. doi:10.1016/j. ijpp.2015.02.003

Yevstafyeva, V. A., Melnychuk, V. V., Nikiforova, O. V., Suprunenko, K. V., Korchan, L. N., Lokes-Krupka, ... Korchan, N. I. (2018). Comparative morphology and biology of nematodes of genus Heterakis (Nematoda, Heterakidae), parasites of the domestic goose (Anser anser) in Ukraine. Regulatory Mechanisms in Biosystems, 9(2), 229-236. doi:10.15421/021834 